4. Proximal jejunal stoma as ultima ratio in case of traumatic distal

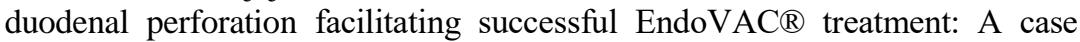
report M. Kelm, F. Seyfried, S. Reimer et al. International Journal of Surgery. 2017; 41:401-403. https://doi.org/10.1016/j.ijscr.2017.11.022

DOI https://doi.org/10.30525/978-9934-26-075-9-6

\title{
LYMPHOID ENVIRONMENT IN TRIPLE-NEGATIVE INVASIVE DUCTAL BREAST CARCINOMA
}

Volos L. I.

Doctor of Medical Science, Professor at the Department of Pathology Danylo Halytsky Lviv National Medical University

\section{Dudash A. P.}

PhD student at the Department of Pathology Danylo Halytsky Lviv National Medical University,

Pathologist

Western Ukrainian Histological Laboratory,

\author{
Guley R. V.
}

MD, Pathologist,

Chair of Western Ukrainian Histological Laboratory

\section{Petronchak O. A.}

$M D$,

Pathologist at the Western Ukrainian Histological Laboratory

Lviv, Ukraine

Introduction. Triple-negative breast cancer (TNBC) is one of molecular subtypes breast cancer, about 10 to 20 percent of women with a breast cancer diagnosis have the triple-negative subtype, tumor doesn't have receptors for the estrogen, progesterone hormones, and doesn't express the protein HER2/neu, doesn't respond to the hormonal therapies and the Herceptin. TNBC has a poor prognosis, with higher grade and tends to spread, grows rapidly and occurs most often in younger women 40 or 50 years old and earlier. One of the promising scientific trends of modern oncology is the study of the role of the immune system in the pathogenesis of malignant tumors 26 
$[1,3,7]$. Scientists shown that the pronounced infiltration of the tumor stroma by immunocompetent cells indicates that the tumor is recognized by the immune system. Some researchers agree that infiltration of breast carcinomas with T-lymphocytes has a positive effect on the prognosis. Some studies have shown a predominance a subpopulation of cytotoxic/suppressor CD8+ cells over the helpers/inducers of CD4+ in malignant tumors of the breast [11].

Today among researchers the concept of tumor infiltrating lymphocytes has been established as a prognosis factor, the so-called TIL factor [tumor infiltrating lymphocytes], its composition, severity and multicomponent $[5,9]$. The reason for this, of course, is the study and attempts to overcome the phenomenon of «evasion by tumor cells of the immune system», and the more pronounced it is in a tumor, the worse the prognosis of the course and metastasis of this tumor. Recent work suggests that it is becoming important to study not only the tumor infiltrating lymphocytes, but also the cellular composition of the zone near to the tumor or peritumoral. Thus, it was shown that the immune response in the tumor was significantly different from that in the adjacent tissue [4]. At the same time, the studies indicate failure of immunelogical surveillance in invasive breast cancer, which is confirmed by the presence of single contacts between cancer and immunocompetent cells, the lack of connections between lymphocytes, macrophages and plasma cells, the absence of this cells in the lumens and perivascular spaces of microvessels. Lymphoid environment plays an important prognostic role in TNBC. The relationship between lymphoid environment and clinical behavior is not fully understood.

The aim of this study was to research the densities of tumor infiltrating lymphocytes in triple-negative breast cancer, and to investigate their associations with prognosis.

Materials and methods. Thirty-two $(16,58 \%)$ TNBC patients were retrospectively selected from a cohort of 193 patients with invasive ductal breast carcinoma diagnosed in 2017 at the Lviv State Oncological Regional Treatment and Diagnostic Center. Clinic data were collected from history cards of disease and patients had undergone surgery treatment without used neoadjuvant chemotherapy. The histological specimens breast cancer were formalin-fixed, paraffin embedded, $5 \pm 1 \mu \mathrm{m}$ thick-section cutted, stained by Hematoxylin-Eosin.

The histologic type of the breast cancer was determined according to the WHO classification. The histologic grade was categorized as grade I, grade II and grade III using the Elston-modified Bloom and Richardson grading system [2]. The TNM was used for clinical staging of breast cancer and clinicopathologic characteristics included tumor size, lymph node status, stage, and 
histologic grades were evaluated. Triple-negative phenotype was analysed for the three markers (ER negative, PR negative, and HER2 negative). ER (Clone EP1, Dako), PR (PgR 636, Dako Flex), Her2-neu (Clone SP3 Thermo scientific) and streptavidin peroxidase detection system with diaminobenzidine tetrachloride were used. Expressions of ER, PR, HER2, tumor-infiltrating lymphocytes were assessed by immunohistochemistry in Western Ukrainian Histological Laboratory (Lviv). Antigen distribution CD3 is expressed on all T-lymphocytes, CD20 is expressed on B-lymphocytes and CD56 is expressed on natural killer (NK) lymphocytes.

Results. The 32 patients with TNBC had a median age of 59 years (range, 31-79 years), $11(34,4 \%)$ were under 55 years. The majority of patients $(43,74 \%)$ were clinical stage II, while III and IV stages were $34,38 \%$ and $12,5 \%$ patients respectively. Nottingham Histologic Grade distribution was as follows: G1 - 0 (0\%), G2 - 17 (53,13\%), and G3 - 14 (43,74\%), G4 - 1 $(3,13 \%)$ cases. Menopausal status was in $65,6 \%$ of cases. A percentage of tumor area was occupied by positively-stained of T- and B-cells. Lymphoid environment intratumoral and at the invasive margin, were significantly higher in G3 carcinoma (CD3 was 10,41 $\pm 0,19$ intratumoral and 35,91 $\pm 1,27$ at the invasive margin; CD20 was 4,56 $\pm 0,14$ and 22,96 $\pm 1,34$ respectively; CD56 was less than 1\%) than G2 (CD3 was 5,23 $\pm 0,17$ intratumoral and 17,37 $\pm 0,25$ at the invasive margin; CD20 was $2,57 \pm 0,08$ and $12,76 \pm 0,19$ respectively; CD56 was $1,12 \pm 0,02$ and 4,32 $\pm 0,13$ respectively also), while the number of CD56 positive cells decreases with the growth of tumor malignancy $(\mathrm{p}<0,01)$.

TILs represent a vital component of the local anti-cancer immune response. The association of tumor-infiltrating lymphocytes in breast cancer with disease outcome has been recognized many years ago [8] and has, more recently, been verified in a number of large studies [14, 6, 10]. Among breast carcinomas, TNBC comprises a distinct disease entity with a unique microenvironment of TILs, and high proliferative activity of the cancer cells $[13,12]$.

CD3 positive lymphocytes infiltrate around tumor cells in areas of active growth of breast carcinoma, in greater numbers are observed in highly malignant tumors. Depending on the increase in the degree of tumor malignancy, the population of CD3 positive cells increased, accumulating in the peritumoral zone of the tumor, around the zones of active tumor growth at the periphery of the tumor, as compared with the intratumoral zones. In the central zones of continuous tumor growth, the number of CD3 lymphocytes was significantly lower than in the zones of active tumor growth.

The comparative characteristic of the severity polymorphic cell infiltrate in the studied zones showed, that B-lymphocytes (CD20-positive) increased in 
the peritumoral zone, sometimes with a tendency to the formation of folliclelike structures without germinal centers and with an increase in the degree of malignant neoplasms, the number of CD20 increased in the peritumoral zone by 2 times at G3 in comparison with degree G2. It was found that with an increase in the degree of malignancy neoplasms, the number of natural killer cells (NK cells, CD56-positive) decreases, both in the intratumoral and peritumoral zones. Moreover, the most pronounced decrease in the representation of these cells was noted by us in the peritumoral zone.

Conclusions. The higher the degree of malignancy of invasive ductal breast carcinoma, the more pronounced the lymphoid infiltration of the peritumoral and intratumoral areas with the accumulation of mainly CD3 positive lymphocytes, CD20 positive lymphocytes, while the number of natural killer cells (CD56 positive cells) decreases with the growth of tumor malignancy.

\section{References:}

1. Ahn SG, Jeong J, Hong S, et al. Current Issues and clinical evidence in tumor-infiltrating lymphocytes in breast cancer. J Pathol Transl Med. 2015; 49: 355-363.

2. Elston, C.W., Ellis, I.O. Pathological prognostic factors in breast cancer. I. The value of histological grade in breast cancer: experience from a large study with long-term follow-up. Histopathology. 1991. 19(5), 403-10. DOI: 10.1111/j.1365-2559. 1991.tb00229.x

3. Harano K, Wang Y, Lim B, Seitz RS, Morris SW, Bailey DB, et al. Rates of immune cell infiltration in patients with triple-negative breast cancer by molecular subtype. PLoS One. 2018. 13(10): e0204513. https://doi.org/10.1371 /journal.pone.0204513

4. Helal TE, Ibrahim E, Alloub AI. Immunohistochemical analysis of tumor-infiltrating lymphocytes in breast carcinoma: relation to prognostic variables. Indian J Pathol Microbiol. 2013; 56: 89-93.

5. Mahmoud SMA, Lee AHS, Paish EC, et al. The prognostic significance of B lymphocytes in invasive carcinoma of the breast. Breast Cancer Res Treat. 2012; 132: 545-553.

6. Mao Y, Qu Q, Chen X, Huang O, Wu J, Shen K. The prognostic value of tumor-infiltrating lymphocytes in breast cancer: a systematic review and meta-analysis. PLoS One. 2016. 11:e0152500. https://doi.org/10.1371/journal.pone.0152500.

7. Matsumoto H, Koo S, Dent R, et al. Role of inflammatory infiltrates in triple negative breast cancer. J Clin Pathol. 2015; 68: 506-510. 
8. Moore OS, Foote FW. The relatively favorable prognosis of medullary carcinoma of the breast. Cancer. 1949. 2:635-642.

9. Salgado R, Denkert C, Demaria S, et al. The evaluation of tumorinfiltrating lymphocytes (TILS) in breast cancer: Recommendations by an International TILS Working Group 2014. Ann Oncol. 2015; 26: 259-271.

10. Savas P, Salgado R, Denkert C, Sotiriou C, Darcy PK, Smyth MJ, Loi S. Clinical relevance of host immunity in breast cancer: from TILs to the clinic. Nat Rev Clin Oncol. 2016. 13:228-241

11. Vihervuori, H., Autere, T.A., Repo, H. et al. Tumor-infiltrating lymphocytes and $\mathrm{CD}^{+} \mathrm{T}$ cells predict survival of triple-negative breast cancer.J Cancer Res Clin Oncol. 2019, 145,3105-3114. https://doi.org/10.1007/s00432-019-03036-5

12. Yang B, Chou J, Tao Y, Wu D, Wu X, Li X, Li Y, Chu Y, Tang F, Shi Y, Ma L, Zhou T, Kaufmann W, Carey LA, Wu J, Hu Z. An assessment of prognostic immunity markers in breast cancer. NPJ Breast Cancer. 2018, 4:35. https://doi.org/10.1038/s41523-018-0088-0

13. $\mathrm{Yu} \mathrm{T}, \mathrm{Di} \mathrm{G}$. Role of tumor microenvironment in triple-negative breast cancer and its prognostic significance. Chin J Cancer Res. 2017, 29:237-252.

14. Yu X, Zhang Z, Wang Z, Wu P, Qiu F, Huang J. Prognostic and predictive value of tumor-infiltrating lymphocytes in breast-cancer: a systematic review and meta-analysis. Clin Transl Oncol. 2016, 18:497-506. 